\title{
Synthesis and Structure-activity Relationship of Antifungal Coniothyriomycin Analogues
}

\author{
KARSTEN KROHN*,a, BrigitTA ELSÄSSER $^{\text {a }}$, SÁNDOR ANTUS ${ }^{b}$, \\ KRISZTina KÓNYA ${ }^{b}$ and EBERHARd AMMERMANN ${ }^{c}$ \\ ${ }^{a}$ Department of Chemistry, University of Paderborn, \\ Warburger Strasse 100, 33098 Paderborn, Germany \\ ${ }^{\mathrm{b}}$ Department of Organic Chemistry, University of Debrecen, \\ Debrecen 4010, Hungary \\ ${ }^{\mathrm{c}}$ BASF AG, Agricultural Center, Crop Protection, Biology, \\ P.O.Box 220, 67114 Limburgerhof, Germany
}

(Received for publication November 16, 2002)

\begin{abstract}
The structure of the antifungal metabolite coniothyriomycin was systematically modified by changing the acids of the open chain imide, modification of the hydrophobicity, variation in the degree of saturation, replacement of carbons by nitrogen or oxygen, and incorporation of the open chain molecule into cyclic arrangements. Structure-activity studies showed that antifungal activity was retained by replacement of phenylacetic acids by benzoic acids in the imide structure but diminished by hydrogenation of the fumaric ester part.
\end{abstract}

In connection with our investigation of fungal metabolites as biologically active agents, we isolated an open chain imide named coniothyriomycin (1) (Fig. 1) with remarkable antifungal activity from an unidentified Coniothyrium fungus ${ }^{1)}$. In this communication, the synthesis of analogues with variation of the substituents on the aromatic ring and their biological activity were also presented. Unfortunately, these open chain mixed amides of phenylacetic and fumaric acid, in spite of excellent shortterm antifungal activity, did not show curative effects. One reason for this was the inherent instability of the imide functionality in the presence of nucleophiles such as water. Therefore, in the hope to increase the chemical stability and retain or even increase antifungal activity, we extended our study to the preparation and biological testing of various analogues by (i) replacement of the substituted phenylacetic acids with substituted benzoic acids, (ii) change of hydrophobicity by variation of the alcohol component, (iii) variation in the degree of saturation of the fumaric acid moiety, (iv) replacement of carbon by nitrogen or oxygen in the middle part of the molecule, and (v) incorporation of the open chain part of the molecule into cyclic arrangements.
Fig. 1. Structure of coniothyriomycin (1).<smiles>COC(=O)/C=C/C(=O)NC(=O)Cc1ccc(Cl)cc1O</smiles>

\section{Results and Discussion}

In the previous syntheses of coniothyriomycin analogues ${ }^{1)}$ we used the method of SUHARA et al. ${ }^{2)}$ starting from nitriles via the corresponding ethyl imidates. However, the yields using this method were poor $(10 \sim 36 \%)$ and the workup of the dark brown reaction mixture was tedious. Our first efforts were therefore directed towards a simplification of the procedure. To that end, equivalent amounts of benzamide (2) were heated with the fumaric monoethyl ester chloride (3a) in toluene (Scheme 1). However, starting with a $1: 1$ ratio, only modest yields of the desired mixed imide 5 resulted and the mixed anhydride 4 was identified as the major side product. Evidently, both theoretically possible reaction pathways a

\footnotetext{
* Corresponding author: kk@chemie.uni-paderborn.de
} 
Scheme 1. Formation of mixed imide 5a starting from amide $\mathbf{2}$ and acid chloride 3a via the mixed anhydride 4 .

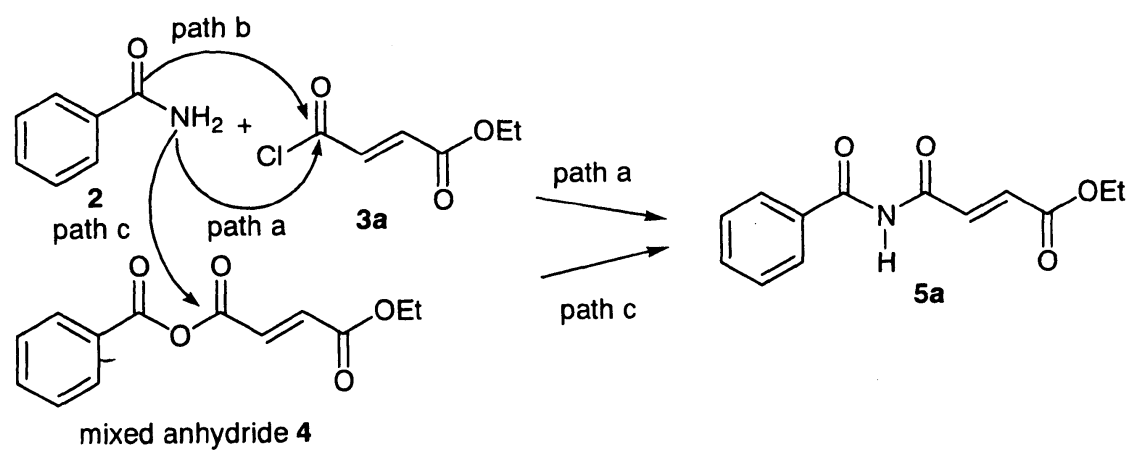

and $b$ were realized: attack by the amide nitrogen on the acyl chloride (path a) to form $\mathbf{5 a}$, and attack by the nucleophilic oxygen (path b) to form 4 . However, the acyl group transfer potential was still preserved in the mixed anhydride $\mathbf{4}$ and attack by excess benzamide (path c) on the mixed anhydride carbonyl might ultimately result in the formation of the mixed open chain imide 5a. In fact, using two equivalents of amide 2, pathway c was also followed consuming the mixed anhydride 4 , and yields of the mixed imide $5 \mathbf{a}$ of $50 \%$ and more were achieved in a clean, easily worked-up reaction. Interestingly, the formation of the symmetric benzoic acid imide, resulting from attack of the nitrogen on the benzoic acid part of anhydride 4, was not observed.

The method proved to be general and most of the mixed imides described in this paper were prepared using this simple procedure. However, in some cases it was advantageous to use the anion of amides such as $\mathbf{2}$. Also, the yields were generally higher starting with benzamide (2) than with phenylacetic acid amide (6a).

The first series of compounds prepared were a number of mixed benzoic acid and fumaric ester open chain imides with variation in the substituents in the aromatic ring and the ester alcohol component. We wanted to see whether omission of the methylene group in 5a by replacement of phenylacetic acid in the coniothriomycin analogues ${ }^{1)}$ with benzoic acid would preserve their antifungal activity. In addition, variation of the substituents from methoxy to fluoride or nitro groups as in $\mathbf{5 b} \sim \mathbf{5 g}$ (Fig. 2) would show the influence of electron density on biological activity. Furthermore, the lipophilicity of the fungicide was increased in $\mathbf{5 h}$ by linking the $n$-octanyl ester of fumaric
Fig. 2. Mixed benzoic acid-fumaric ester imides $5 \mathbf{a} \sim 5 \mathbf{h}$<smiles>[R7]c1cc(C(=O)NC(=O)/C=C/C(=O)OP)c([R])c([R])c1[2H]</smiles>

acid chloride to benzamide. The data and substituents of the mixed benzoic acid-fumaric ester imides $\mathbf{5 a} \sim \mathbf{5} \mathbf{h}$ are listed in Table 1.

The next task was the variation in the degree of saturation in the fumaric ester part, starting from phenylacetic amide as well as benzoic acid amide. Omission of a methine group, e.g. the shift from fumaric ester to malonic ester, or replacement with benzoic acid (incorporating the double bond of fumaric acid into a ring system) was also in line with this type of variation and worth testing for fungicidal activity. The construction of the mixed imides $10 \sim 14$ by coupling of amides 2 and $6 \mathbf{a}, \mathbf{b}$ with the acid chlorides $\mathbf{7 \sim 9}$ is shown in Scheme 2, employing about two equivalents of the respective amides as outlined in Scheme 1. The phenolic imide 11c, as present in the natural product coniothyriomycin (1), was prepared by reaction of benzyl ether $\mathbf{6 b}$ with 7 , followed by hydrogenolysis of benzyl ether 11b to 11c in order to evaluate the influence of a phenolic hydroxy group on activity.

Next, we investigated the replacement of carbon by 
Table 1. Data and substituents of mixed benzoic acid-fumaric ester imides $\mathbf{5 a} \sim \mathbf{5 h}$

\begin{tabular}{llllllll}
\hline & $\mathrm{R}^{1}$ & $\mathrm{R}^{2}$ & $\mathrm{R}^{3}$ & $\mathrm{R}^{4}$ & $\mathrm{R}^{5}$ & $\mathrm{mp}\left({ }^{\circ} \mathrm{C}\right)$ & $\begin{array}{l}\text { yield } \\
(\%)\end{array}$ \\
\hline $\mathbf{5 a}$ & $\mathrm{H}$ & $\mathrm{H}$ & $\mathrm{H}$ & $\mathrm{H}$ & $\mathrm{C}_{2} \mathrm{H}_{5}$ & $87-89^{\circ} \mathrm{C}$ & $51 \%$ \\
$\mathbf{5 b}$ & $\mathrm{F}$ & $\mathrm{H}$ & $\mathrm{H}$ & $\mathrm{H}$ & $\mathrm{C}_{2} \mathrm{H}_{5}$ & $54-55^{\circ} \mathrm{C}$ & $42 \%$ \\
$\mathbf{5 c}$ & $\mathrm{H}$ & $\mathrm{H}$ & $\mathrm{NO}_{2}$ & $\mathrm{H}$ & $\mathrm{C}_{2} \mathrm{H}_{5}$ & $135-137^{\circ} \mathrm{C}$ & $44 \%$ \\
$\mathbf{5 d}$ & $\mathrm{OCH}_{3}$ & $\mathrm{H}$ & $\mathrm{H}$ & $\mathrm{H}$ & $\mathrm{C}_{2} \mathrm{H}_{5}$ & $98-100^{\circ} \mathrm{C}$ & $46 \%$ \\
$\mathbf{5 e}$ & $\mathrm{H}$ & $\mathrm{H}$ & $\mathrm{OCH}_{3}$ & $\mathrm{H}$ & $\mathrm{C}_{2} \mathrm{H}_{5}$ & $120-122^{\circ} \mathrm{C}$ & $45 \%$ \\
$\mathbf{5 f}$ & $\mathrm{H}$ & $\mathrm{OCH}_{3}$ & $\mathrm{H}$ & $\mathrm{OCH}_{3}$ & $\mathrm{C}_{2} \mathrm{H}_{5}$ & $162-164^{\circ} \mathrm{C}$ & $43 \%$ \\
$\mathbf{5 g}$ & $\mathrm{H}$ & $\mathrm{OCH}_{3}$ & $\mathrm{OCH}$ & $\mathrm{OCH}_{3}$ & $\mathrm{C}_{2} \mathrm{H}_{5}$ & $114-116^{\circ} \mathrm{C}$ & $65 \%$ \\
$\mathbf{5 h}$ & $\mathrm{H}$ & $\mathrm{H}$ & $\mathrm{H}$ & $\mathrm{H}$ & $n-\mathrm{C}_{8} \mathrm{H}_{15}$ & oil & $33 \%$ \\
\hline
\end{tabular}

Scheme 2. Construction of mixed imides $\mathbf{1 0} \sim \mathbf{1 4}$ by coupling of amides 2 and $\mathbf{6 a}, \mathbf{b}$ with the acid chlorides 7 9.<smiles>[R]c1ccc(CC(N)=O)cc1</smiles>

a: $R=H, b: R=O B n, c: R=O H$<smiles>COC(=O)CCC(=O)NC(=O)Cc1ccccc1</smiles><smiles>[R]OC(=O)CCC(=O)N(C)C(=O)Cc1ccc(C(=O)NC(=O)CC(=O)OC)cc1</smiles>

12<smiles>[R]C1CC=CC(C(=O)NC(=O)Cc2ccccc2)=CC1</smiles>

13: $R=3-\mathrm{OCH}_{3}$ 14: $\mathrm{R}=4-\mathrm{OCH}_{3}$

with diethyl oxalate $(\mathbf{2 1})^{3)}$. The carbonyl group of 23 was then reacted with a number of Wittig ylides to afford the E-olefins 24 27 (compare ${ }^{4)}$ ).

\section{Biological Studies}

The tested synthetic coniothyriomycin analogues showed primarily control of plant diseases caused by representative fungi belonging to the class of Oomycetes, e.g. late blight on tomatoes caused by Phytophthora infestans or downy mildew on grape vine caused by Plasmopara viticola. The fungicidal activity was tested in vitro in 96-well microtitre plates and on intact plants in a greenhouse.

The change of the molecule fragment phenylacetic amide 
Scheme 3. Synthesis of bis-hydrazide 16, acyl hydroxamic acid $\mathbf{1 8}$ and fumaric hydrazides $\mathbf{2 0 a} \sim \mathbf{c}$.<smiles>NNC(=O)c1ccccc1</smiles>

15

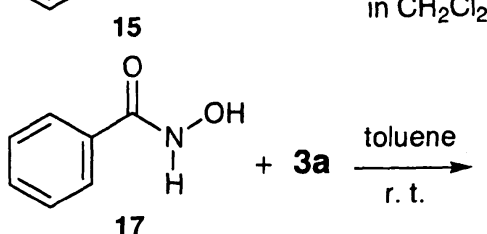<smiles>NNc1ccccc1</smiles>

a: $R=H, R^{\prime}=E t$

b: $R=2,4-d i-N_{2}, R^{\prime}=E t$

c: $\mathrm{R}=\mathrm{H}, \mathrm{R}^{\prime}=n-\mathrm{C}_{8} \mathrm{H}_{15}$<smiles>CCOC(=O)/C=C/C(=O)NNC(=O)c1ccccc1</smiles>

16<smiles>CCOC(=O)/C=C/C(=O)ONC(=O)c1ccccc1</smiles>

18<smiles>[R]c1ccc(NN(NC(=O)/C=C/C(=O)OCC)C(C)=O)cc1</smiles>

20a-c<smiles>CCOC(=O)/C=C/C(=O)Cl</smiles>

Scheme 4. Wittig reaction of 4-phenyl-pyrrolidine-2,3,5-trione (23) to yield the $E$-olefins $24 \sim 27$.

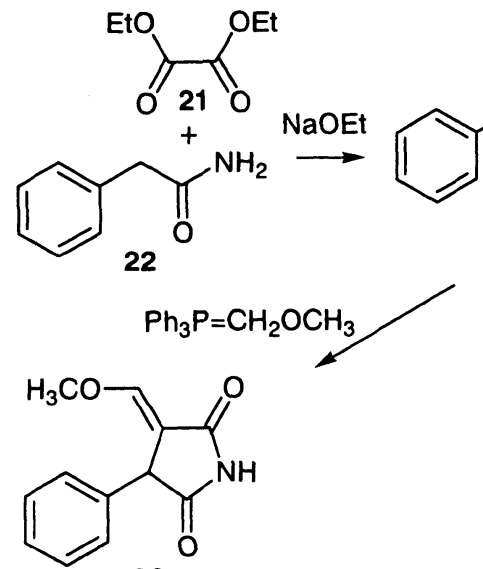

26

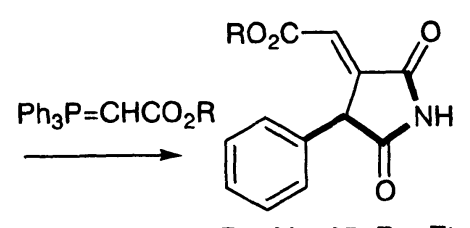

24: $R=M e, 25: R=E t$<smiles>CCOC(=O)CC=C1C(=O)NC(=O)C1c1ccccc1</smiles>

27 to benzoic amide in $\mathbf{5 a}$ to $\mathbf{5 h}$ retained the same good in vitro activity as the lead structure coniothyriomycin. 5a to 5d controlled $P$. infestans with $\mathrm{ED}_{90}$-values of less than $0.5 \mathrm{ppm}, 5 \mathrm{e}$ even with less than $0.125 \mathrm{ppm}$. Some additional control of the causal organism of rice blast, Pyricularia oryzae, and of the causal organism of leaf blotch on wheat, Septoria tritici was observed for $\mathbf{5 a}$ to $5 \mathbf{e}$ with $\mathrm{ED}_{90}$-values of less than 2 and $8 \mathrm{ppm}$ respectively. The fungicidal in vitro activity could only partially be translated into in vivo activity in greenhouse tests on intact plants. Only $\mathbf{5 f}$ showed some initial protective control of $P$. infestans on tomatoes with additional initial good protective activity against $P$. viticola on grapes. The compounds $\mathbf{5 d}, \mathbf{5 g}$ and $\mathbf{5 h}$ showed only some moderate control of $P$. viticola.

The hydrogenation of the double bond in the fumaric acid fragment, which resulted in 10 and $11 \mathbf{a}$, led to total loss of fungicidal activity both in vitro and in vivo. The other structural variations in the compounds 13 an 14 
resulted in reduced in vitro fungicidal activity against $P$. infestans, with $\mathrm{ED}_{90}$-values of less than 31 and $8 \mathrm{ppm}$ respectively. All other structural variations in the compounds 12, 16, 18, 20a $\sim$ c and 24 led to a loss of any significant in vitro or in vivo fungicidal activity.

\section{Experimental}

\section{Biological Tests}

The in vitro tests were run in 96-well microtitre plates. The wells were filled with aqueous solutions of the compounds in the appropriate concentration prepared from a DMSO-stock solution. Thereafter the spore suspensions were added. The spores of Botrytis cinerea, Pyricularia oryzae and Septori tritici were suspended in aqueous malt extract at a final concentration of $2 \%$, the spores of Phytophthora infestans in a $2 \%$ aqueous synthetic medium according to SCHEEPENS and FEHRMANN ${ }^{5)}$.

The plates were kept for 7 days at $18^{\circ} \mathrm{C}$ and constant humidity. Mycelial growth was assessed with a THERMOmax microplate reader from Molecular Devices at $405 \mathrm{~nm}$ and the values were related to the untreated check.

The in vivo tests were done on intact plants in a greenhouse. The plants were sprayed to run-off with an aqueous solution or suspension of the compound. The airdried plants were inoculated the following day with aqueous spore suspensions of the appropriate fungus. The inoculated plants were then kept in growth chambers with high humidity and at temperatures favourable for the development of the plant disease. After 4 to 7 days, the disease development on the untreated checks had almost covered the whole leaf area. At this point in time the trials were assessed.

\section{Chemical Synthesis}

For general methods and instrumentation $\operatorname{see}^{6}$.

Condensation of Amides with Acid Chlorides, General Procedure $\mathrm{A}$ and $\mathrm{B}$

Procedure A: To a boiling solution of the amide (10 $\mathrm{mmol})$ in dry toluene $(20 \mathrm{ml})$ a solution of the acid chloride $(5 \mathrm{mmol})$ in toluene $(5 \mathrm{ml})$ was added dropwise. The mixture was refluxed overnight (TLC monitoring), the solvent was removed under reduced pressure and the residue was purified by column chromatography on silica gel (dichloromethane) and recrystallized from ether. For yields and $\mathrm{mp}$ of $\mathbf{5 a} \sim \mathbf{5 h}$ see Table 1 .

Procedure B: Alternatively, the sodium salt of the amide (prepared by reaction of the amide solution with sodium hydride) was employed, with fumaric acid monoethyl ester anhydride.

\section{4-Benzoylamino-4-oxobut-2-enoic Acid Ethyl Ester (5a)}

Benzamide (2) $(1.23 \mathrm{~g}, 10 \mathrm{mmol})$ in dry toluene $(20 \mathrm{ml})$ was reacted according to procedure $A$ with fumaric acid monoethyl ester chloride (3a) $(1.78 \mathrm{~g}, 7.5 \mathrm{mmol})$ in toluene $(5 \mathrm{ml})$ to yield $\mathbf{5 a}(1.18 \mathrm{~g}, 51 \%)$, mp: $87 \sim 89^{\circ} \mathrm{C}$. IR $(\mathrm{KBr})$ $\tilde{v}=3291 \mathrm{~cm}^{-1}(\mathrm{~N}-\mathrm{H}), 1724(\mathrm{C}=\mathrm{O}), 1703 \quad(\mathrm{C}=\mathrm{O}), 1672$ $(\mathrm{C}=\mathrm{O}) .{ }^{1} \mathrm{H}$ NMR $\left(200 \mathrm{MHz}, \mathrm{CDCl}_{3}\right): \delta=1.36(\mathrm{t}, J=7 \mathrm{~Hz}$, $\left.3 \mathrm{H}, \mathrm{CH}_{3}\right), 4.32$ (q, J=7 Hz, 2H, $\left.\mathrm{CH}_{2}\right), 6.90$ (d, $J=2 \mathrm{~Hz}, 2 \mathrm{H}$, $\mathrm{CH}), 7.02$ (d, $J=2 \mathrm{~Hz}, 2 \mathrm{H}, \mathrm{CH}), 7.61$ (m, 3H, ArH), 7.96 $(\mathrm{m}, 2 \mathrm{H}, 5 \mathrm{ArH}), 9.43(\mathrm{~s}, 1 \mathrm{H}, \mathrm{NH}) .{ }^{13} \mathrm{C} \mathrm{NMR}(50 \mathrm{MHz}$, $\left.\mathrm{CDCl}_{3}\right): \delta=14.6\left(\mathrm{CH}_{3}\right), 61.8\left(\mathrm{CH}_{2}\right), 128.5(\mathrm{CH}), 129.4$ (CH), 133.3 (ArC), 134.1 (ArC), 134.3 (ArC), 135.2 (ArC), $165.4(\mathrm{C}=\mathrm{O}), 166.3(\mathrm{C}=\mathrm{O}), 167.3(\mathrm{C}=\mathrm{O})$. Anal Calcd for $\mathrm{C}_{12} \mathrm{H}_{11} \mathrm{NO}_{4}$ (233.22): C 63.09, H 5.26; Found: C 61.66, H 5.29 .

4-(2-Fluorobenzoylamino)-4-oxobut-2-enoic Acid Ethyl Ester (5b)

2-Fluorobenzoic acid amide $(2.000 \mathrm{~g}, 14.4 \mathrm{mmol})$ in dry toluene $(15 \mathrm{ml})$ was reacted with fumaric acid chloride (3a) $(1.300 \mathrm{~g}, 9.6 \mathrm{mmol})$ as described in procedure A to afford imide 5b $(890 \mathrm{mg}, 42 \%), \mathrm{mp}: 54 \sim 55^{\circ} \mathrm{C}$. IR $(\mathrm{KBr})$ $\tilde{v}=3388 \mathrm{~cm}^{-1}(\mathrm{~N}-\mathrm{H}), 1720 \quad(\mathrm{C}=\mathrm{O}), 1705 \quad(\mathrm{C}=\mathrm{O}), 1678$ $(\mathrm{C}=\mathrm{O}) .{ }^{1} \mathrm{H}$ NMR $\left(200 \mathrm{MHz}, \mathrm{CDCl}_{3}\right): \delta=1.35(\mathrm{t}, J=7 \mathrm{~Hz}$, $\left.3 \mathrm{H}, \mathrm{CH}_{3}\right), 4.30\left(\mathrm{q}, J=7 \mathrm{~Hz}, 2 \mathrm{H}, \mathrm{CH}_{2}\right), 6.94(\mathrm{~d}, J=14 \mathrm{~Hz}$, $1 \mathrm{H}, \mathrm{CH}), 7.38$ (m, 2H, ArH), 7.61 (m, 1H, ArH), 7.88 (d, $J=14 \mathrm{~Hz}, 1 \mathrm{H}, \mathrm{CH}), 8.08$ (m, 1H, ArH), 9.19 (d, $J=13 \mathrm{~Hz}$, $1 \mathrm{H}, \mathrm{NH}) .{ }^{13} \mathrm{C} \mathrm{NMR}\left(50 \mathrm{MHz}, \mathrm{CDCl}_{3}\right): \delta=14.5\left(\mathrm{CH}_{3}\right), 61.8$ $\left(\mathrm{CH}_{2}\right), 116.9(\mathrm{CH}), 120.4(\mathrm{ArC}), 125.8(\mathrm{CH}), 132.7$ (ArC), 134.7 (ArC), 135.4 (ArC), 135.8 (ArC), $158.5(\mathrm{C}=\mathrm{O})$, $162.5(\mathrm{C}=\mathrm{O}), 163.5(\mathrm{C}=\mathrm{O}), 165.6$ (ArC). Anal Calcd for $\mathrm{C}_{13} \mathrm{H}_{12} \mathrm{FNO}_{4}$ (265.24): C 58.87, $\mathrm{H} 4.56, \mathrm{~N} 5.28$. Found: $\mathrm{C}$ 58.39, H 4.46, N 5.24.

4-(4-Nitrobenzoylamino)-4-oxobut-2-enoic Acid Ethyl Ester (5c)

4-Nitrobenzoic acid amide (550 $\mathrm{mg}, 3.3 \mathrm{mmol})$ was reacted with fumaric acid chloride (3a) $(200 \mathrm{mg}, 1.5 \mathrm{mmol})$ as described in procedure $A$ to afford imide $5 \mathbf{c}(158 \mathrm{mg}$, $44 \%$ ), mp: $135 \sim 137^{\circ} \mathrm{C}$. IR (KBr) $\tilde{v}=3244 \mathrm{~cm}^{-1}(\mathrm{~N}-\mathrm{H})$, $1720(\mathrm{C}=\mathrm{O}), 1713(\mathrm{C}=\mathrm{O}), 1678 \quad(\mathrm{C}=\mathrm{O}) .{ }^{1} \mathrm{H} \quad \mathrm{NMR}$ (200 MHz, DMSO): $\delta=1.28\left(\mathrm{t}, J=7 \mathrm{~Hz}, 3 \mathrm{H}, \mathrm{CH}_{3}\right), 4.24(\mathrm{q}$, $\left.J=7 \mathrm{~Hz}, 2 \mathrm{H}, \mathrm{CH}_{2}\right), 6.75$ (d, $\left.J=16 \mathrm{~Hz}, 1 \mathrm{H}, \mathrm{CH}\right), 7.54$ (d, $J=16 \mathrm{~Hz}, 1 \mathrm{H}, \mathrm{CH}), 8.15$ (d, $J=9 \mathrm{~Hz}, 2 \mathrm{H}, \mathrm{ArH}), 8.37$ (d, $J=9 \mathrm{~Hz}, 2 \mathrm{H}, \mathrm{ArH}), 11.73(\mathrm{~s}, 1 \mathrm{H}, \mathrm{NH}) .{ }^{13} \mathrm{C} \mathrm{NMR}(50 \mathrm{MHz}$, DMSO): $\delta=14.8\left(\mathrm{CH}_{3}\right), 59.6\left(\mathrm{CH}_{2}\right), 134.4(\mathrm{CH}), 137.8$ 
(CH), 123.7 (ArC), 123.8 (ArC), 128.2 (ArC), 128.3 (ArC), 139.4 (ArC), 150.6 (ArC), $165.1(\mathrm{C}=\mathrm{O}), 165.5(\mathrm{C}=\mathrm{O})$, $166.4(\mathrm{C}=\mathrm{O})$. Anal Calcd for $\mathrm{C}_{13} \mathrm{H}_{12} \mathrm{~N}_{2} \mathrm{O}_{6}(292.24)$ : $\mathrm{C}$ 53.43, H 4.14, N 9.59; Found: C: 55.29, H 4.53, N 8.56.

\section{4-(2-Methoxybenzoylamino)-4-oxobut-2-enoic Acid Ethyl}

\section{Ester (5d)}

2-Methoxybenzoic acid amide $(2.100 \mathrm{~g}, 13.9 \mathrm{mmol})$ was reacted with fumaric acid chloride (3a) $(1.000 \mathrm{~g}, 7.4 \mathrm{mmol})$ as described in procedure $A$ to afford imide $\mathbf{5 d}(785 \mathrm{mg}$, $46 \%$ ), $\mathrm{mp}: \quad 98 \sim 100^{\circ} \mathrm{C}$. IR $(\mathrm{KBr}) \tilde{v}=3303 \mathrm{~cm}^{-1}(\mathrm{~N}-\mathrm{H})$, $1720(\mathrm{C}=\mathrm{O}), 1705(\mathrm{C}=\mathrm{O}), 1684(\mathrm{C}=\mathrm{O}) .{ }^{1} \mathrm{H}$ NMR $(200$ $\left.\mathrm{MHz}, \mathrm{CDCl}_{3}\right): \delta=1.38\left(\mathrm{t}, J=7 \mathrm{~Hz}, 3 \mathrm{H}, \mathrm{CH}_{3}\right), 4.09(\mathrm{~s}, 3 \mathrm{H}$, $\left.\mathrm{OCH}_{3}\right), 4.32$ (q, J=7 Hz, 2H, $\mathrm{CH}_{2}$ ), $6.94(\mathrm{~d}, J=14 \mathrm{~Hz}, 1 \mathrm{H}$, $\mathrm{CH}), 7.18(\mathrm{~m}, 2 \mathrm{H}, \mathrm{ArH}), 7.62(\mathrm{~m}, 1 \mathrm{H}, \mathrm{ArH}), 8.00(\mathrm{~d}$, $J=14 \mathrm{~Hz}, 1 \mathrm{H}, \mathrm{CH}), 8.24(\mathrm{~m}, 1 \mathrm{H}, \mathrm{ArH}), 10.44$ (s, 1H, NH). ${ }^{13} \mathrm{C}$ NMR $\left(50 \mathrm{MHz}, \mathrm{CDCl}_{3}\right): \delta=14.5\left(\mathrm{CH}_{3}\right), 56.7\left(\mathrm{OCH}_{3}\right)$, $61.7\left(\mathrm{CH}_{2}\right), 112.2(\mathrm{CH}), 120.1(\mathrm{ArC}), 122.2(\mathrm{CH}), 133.3$ (ArC), 135.6 (ArC), 136.3 (ArC), 136.9 (ArC), 158.2 (ArC), $164.2(\mathrm{C}=\mathrm{O}), 165.6(\mathrm{C}=\mathrm{O}), 166.4(\mathrm{C}=\mathrm{O})$. Anal Calcd for $\mathrm{C}_{14} \mathrm{H}_{15} \mathrm{NO}_{5}$ (277.27): C 60.64, H 5.45, N 5.05; Found: C 60.38, H 5.47, N 5.19.

4-(4-Methoxybenzoylamino)-4-oxobut-2-enoic Acid Ethyl Ester $(\mathbf{5 e})$

4-Methoxybenzoic acid amide (500 $\mathrm{mg}, 3.3 \mathrm{mmol}$ ) in dry toluene $(8 \mathrm{ml})$ was reacted with fumaric acid chloride (3) $(240 \mathrm{mg}, 1.8 \mathrm{mmol})$ as described in A to afford $\mathbf{5 e}(185 \mathrm{mg}$, $45 \%$ ), mp: $120 \sim 122^{\circ} \mathrm{C}$. IR $(\mathrm{KBr}) \tilde{v}=3271 \mathrm{~cm}^{-1}(\mathrm{~N}-\mathrm{H})$, $1726(\mathrm{C}=\mathrm{O}), 1709(\mathrm{C}=\mathrm{O}), 1668(\omega \mathrm{C}=\mathrm{O}) .{ }^{1} \mathrm{H}$ NMR $\left(200 \mathrm{MHz}, \mathrm{CDCl}_{3}\right): \delta=1.38\left(\mathrm{t}, J=7 \mathrm{~Hz}, 3 \mathrm{H}, \mathrm{CH}_{3}\right), 3.93(\mathrm{~s}$, $\left.3 \mathrm{H}, \mathrm{OCH}_{3}\right), 4.32\left(\mathrm{q}, J=7 \mathrm{~Hz}, 2 \mathrm{H}, \mathrm{CH}_{2}\right), 6.98(\mathrm{~d}, J=14 \mathrm{~Hz}$, $1 \mathrm{H}, \mathrm{CH}), 7.03$ (d, $J=8 \mathrm{~Hz}, 2 \mathrm{H}, \mathrm{ArH}), 7.89$ (d, $J=8 \mathrm{~Hz}, 2 \mathrm{H}$, $\mathrm{ArH}), 8.04$ (d, $J=14 \mathrm{~Hz}, 1 \mathrm{H}, \mathrm{CH}), 8.70$ (s, 1H, NH). ${ }^{13} \mathrm{C}$ $\operatorname{NMR}\left(50 \mathrm{MHz}, \mathrm{CDCl}_{3}\right): \delta=14.5\left(\mathrm{CH}_{3}\right), 55.9\left(\mathrm{OCH}_{3}\right), 61.7$ $\left(\mathrm{CH}_{2}\right), 114.6(\mathrm{CH}), 124.6(\mathrm{ArC}), 130.8(\mathrm{CH}), 133.8(\mathrm{ArC})$, 135.4 (ArC), 136.7 (ArC), 137.5 (ArC), 164.4 (ArC), 165.5 $(\mathrm{C}=\mathrm{O}), 165.7(\mathrm{C}=\mathrm{O}), 167.5(\mathrm{C}=\mathrm{O})$. Anal Calcd for $\mathrm{C}_{14} \mathrm{H}_{15} \mathrm{NO}_{5}$ (277.27): C 60.64, H 5.45, N 5.05; Found: C: 59.97, H: 5.62, N 5.22.

4-(3,5-Dimethoxybenzoylamino)-4-oxobut-2-enoic Acid Ethyl Ester (5f)

3,4-Dimethoxy-benzoic acid amide (500 mg, $2.8 \mathrm{mmol}$ ) in dry toluene $(8 \mathrm{ml})$ was reacted with fumaric acid chloride (3a) $(200 \mathrm{mg}, 1.5 \mathrm{mmol})$ as described in procedure $\mathrm{A}$ to afford $5 f(362 \mathrm{mg}, 43 \%), \mathrm{mp}: 162 \sim 164^{\circ} \mathrm{C}$. IR (KBr) $\tilde{v}=3271 \mathrm{~cm}^{-1}(\mathrm{~N}-\mathrm{H}), 1722(\mathrm{C}=\mathrm{O}), 1705(\mathrm{C}=\mathrm{O}), 1675$ $(\mathrm{C}=\mathrm{O}) .{ }^{1} \mathrm{H}$ NMR $\left(200 \mathrm{MHz}, \mathrm{CDCl}_{3}\right): \delta=1.38(\mathrm{t}, J=7 \mathrm{~Hz}$, $\left.3 \mathrm{H}, \mathrm{CH}_{3}\right), 3.88\left(\mathrm{~s}, 6 \mathrm{H}, 2 \times \mathrm{OCH}_{3}\right), 4.33(\mathrm{q}, J=7 \mathrm{~Hz}, 2 \mathrm{H}$,
$\left.\mathrm{CH}_{2}\right), 6.72(\mathrm{~d}, J=14 \mathrm{~Hz}, 1 \mathrm{H}, \mathrm{CH}), 7.01(\mathrm{~s}, 1 \mathrm{H}, \mathrm{ArH}), 7.30$ (d, $J=14 \mathrm{~Hz}, 1 \mathrm{H}, \mathrm{CH}), 8.02$ (d, $J=8 \mathrm{~Hz}, 2 \mathrm{H}, \operatorname{ArH}), 8.78$ (s, $1 \mathrm{H}, \mathrm{NH}) \cdot{ }^{13} \mathrm{C} \mathrm{NMR}\left(50 \mathrm{MHz}, \mathrm{CDCl}_{3}\right): \delta=14.5\left(\mathrm{CH}_{3}\right), 56.1$ $\left(\mathrm{OCH}_{3}\right), 61.6\left(\mathrm{OCH}_{2}\right), 112.6(\mathrm{CH}), 124.8(\mathrm{ArC}), 130.4$ (CH), 133.2 (ArC), 136.1 (ArC), 136.8 (ArC), 137.9 (ArC), 164.4 (ArC), $165.8(\mathrm{C}=\mathrm{O}), 166.1(\mathrm{C}=\mathrm{O}), 167.3(\mathrm{C}=\mathrm{O})$. Anal Calcd for $\mathrm{C}_{15} \mathrm{H}_{17} \mathrm{NO}_{6}$ (307.30): C 58.63, H 5.58, N 4.56. Found: C 59.77, H 5.52.

4-(3,4,5-Trimethoxybenzoylamino)-4-oxobut-2-enoic Acid Ethyl Ester (5g)

The sodium salt of the amide was prepared by stirring a mixture 3,4,5-trimethoxy-benzoic acid amide (4 g, 18.9 $\mathrm{mmol})$ and $\mathrm{NaH}(1 \mathrm{~g}, 20.8 \mathrm{mmol})$ in THF $(100 \mathrm{ml})$ for 30 minutes, and reacted with fumaric acid monoethyl ester anhydride $(5.1 \mathrm{~g}, 18.9 \mathrm{mmol})$ in $30 \mathrm{ml}$ THF (procedure B). After column chromatography, $\mathbf{5 g}$ was isolated $(4.1 \mathrm{~g}$, $65 \%)$ mp: $114 \sim 116^{\circ} \mathrm{C}$. IR $(\mathrm{KBr}) \tilde{v}=3255 \mathrm{~cm}^{-1}(\mathrm{~N}-\mathrm{H})$, $1729(\mathrm{C}=\mathrm{O}), 1707(\mathrm{C}=\mathrm{O}), 1672(\mathrm{C}=\mathrm{O}) .{ }^{1} \mathrm{H}$ NMR $(200$ $\left.\mathrm{MHz}, \mathrm{CDCl}_{3}\right): \delta=1.38\left(\mathrm{t}, J=7 \mathrm{~Hz}, 3 \mathrm{H}, \mathrm{CH}_{3}\right), 3.97(\mathrm{~s}, 9 \mathrm{H}$, $\left.3 \times \mathrm{OCH}_{3}\right), 4.32\left(\mathrm{q}, J=7 \mathrm{~Hz}, 2 \mathrm{H}, \mathrm{CH}_{2}\right), 6.94(\mathrm{~d}, J=14 \mathrm{~Hz}$, $1 \mathrm{H}, \mathrm{CH}), 7.21$ (s, 2H, ArH), 8.05 (d, $J=14 \mathrm{~Hz}, 1 \mathrm{H}, \mathrm{CH})$, $9.42(\mathrm{~s}, 1 \mathrm{H}, \mathrm{NH}) .{ }^{13} \mathrm{C}$ NMR $\left(50 \mathrm{MHz}, \mathrm{CDCl}_{3}\right): \delta=14.5$ $\left(\mathrm{CH}_{3}\right), 56.6\left(\mathrm{OCH}_{3}\right), 56.7\left(\mathrm{OCH}_{3}\right), 61.3\left(\mathrm{OCH}_{3}\right), 61.9$ $\left(\mathrm{OCH}_{2}\right), 106.2(\mathrm{CH}), 124.7(\mathrm{ArC}), 127.1(\mathrm{CH}), 133.6$ (ArC), 135.3 (ArC), 143.2 (ArC), 153.3 (ArC), 165.2 (ArC), $166.0(\mathrm{C}=\mathrm{O}), 168.5(\mathrm{C}=\mathrm{O}), 169.2(\mathrm{C}=\mathrm{O})$. Anal Calcd for $\mathrm{C}_{16} \mathrm{H}_{19} \mathrm{NO}_{7}$ (337.32): C 56.97, H 5.68. Found: $\mathrm{C}$ 56.91, H 6.02 .

\section{4-Benzoylamino-4-oxobut-2-enoic Acid Octyl Ester (5h)}

The sodium salt of benzamide was prepared by stirring a mixture of benzamide (500 mg, $4.1 \mathrm{mmol})$ and $\mathrm{NaH}(172$ $\mathrm{mg}, 4.1 \mathrm{mmol})$ in THF $(35 \mathrm{ml})$ for 30 minutes, and reacted with fumaric acid monooctyl ester anhydride $(1.82 \mathrm{~g}, 4.1$ $\mathrm{mmol}$ ) in $15 \mathrm{ml}$ THF (procedure B). After column chromatography, 13 was isolated as an oil (190 mg, 13\%). IR $(\mathrm{KBr}) \tilde{v}=3292 \mathrm{~cm}^{-1}(\mathrm{~N}-\mathrm{H}), 1724(\mathrm{C}=\mathrm{O}), 1709(\mathrm{C}=\mathrm{O})$, $1687(\mathrm{C}=\mathrm{O}) .{ }^{1} \mathrm{H}$ NMR $\left(200 \mathrm{MHz}, \mathrm{CDCl}_{3}\right): \delta=0.91(\mathrm{t}$, $\left.J=6 \mathrm{~Hz}, 3 \mathrm{H}, \mathrm{CH}_{3}\right), 1.31\left(\mathrm{~m}, 10 \mathrm{H}, \mathrm{CH}_{2}\right), 4.25(\mathrm{q}, J=3 \mathrm{~Hz}$, $\left.2 \mathrm{H}, \mathrm{CH}_{2}\right), 6.95$ (d, J=14 Hz, 1H, CH), 7.55 (m, 2H, ArH), $7.68(\mathrm{~m}, 1 \mathrm{H}, \mathrm{ArH}), 7.98$ (m, 2H, ArH), 8.06 (d, $J=14 \mathrm{~Hz}$, $1 \mathrm{H}, \mathrm{CH}), 9.02(\mathrm{~s}, 1 \mathrm{H}, \mathrm{NH}) .{ }^{13} \mathrm{C} \mathrm{NMR}\left(50 \mathrm{MHz}, \mathrm{CDCl}_{3}\right)$ : $\delta=14.5\left(\mathrm{CH}_{3}\right), 23.1\left(\mathrm{CH}_{2}\right), 26.3\left(\mathrm{CH}_{2}\right), 28.9\left(\mathrm{CH}_{2}\right), 29.6$ $\left(\mathrm{CH}_{2}\right), 32.2\left(\mathrm{CH}_{2}\right), 66.1\left(\mathrm{OCH}_{2}\right), 112.6(\mathrm{CH}), 128.4(\mathrm{ArC})$, $129.4(\mathrm{CH}), 132.6$ (ArC), 134.0 (ArC), 134.3 (ArC), 135.2 (ArC), 165.6 (ArC), $166.3(\mathrm{C}=\mathrm{O}), 166.7(\mathrm{C}=\mathrm{O}), 167.0$ $(\mathrm{C}=\mathrm{O})$. Anal Calcd for $\mathrm{C}_{19} \mathrm{H}_{25} \mathrm{NO}_{4}$ (331.41): $\mathrm{C} 68.86, \mathrm{H}$ 7.60; Found: C 69.67, H 7.51. 
4-Oxo-4-phenylacetylaminobutyric Acid Methyl Ester (10)

Phenylacetic acid amide (6a) $(1.35 \mathrm{~g}, 10 \mathrm{mmol})$ in dry toluene $(20 \mathrm{ml})$ was reacted with succinic acid monomethyl ester chloride $(7)^{7)}(1.65 \mathrm{~g}, 12.26 \mathrm{mmol})$ as described in general procedure A to afford $692 \mathrm{mg}(28 \%)$ of 10 . IR $(\mathrm{KBr}): \tilde{v}=3260 \mathrm{~cm}^{-1}, 1735(\mathrm{C}=\mathrm{O}), 1705(\mathrm{C}=\mathrm{O}), 1657$ $(\mathrm{C}=\mathrm{O}) .{ }^{1} \mathrm{H}$ NMR $\left(200 \mathrm{MHz}, \mathrm{CDCl}_{3}\right): \delta=2.69(\mathrm{t}, J=2 \mathrm{~Hz}$, $4 \mathrm{H}, \mathrm{CH}_{2}$ ), 3.69 (s, $2 \mathrm{H}, \mathrm{PhCH}_{2}$ ), 3.70 (s, 3H, $\left.\mathrm{OCH}_{3}\right), 7.37$ $(\mathrm{m}, 5 \mathrm{H}, \mathrm{ArH}), 9.05(\mathrm{~s}, 1 \mathrm{H}, \mathrm{NH}) .{ }^{13} \mathrm{C} \mathrm{NMR}(50 \mathrm{MHz}$, $\left.\mathrm{CDCl}_{3}\right): \delta=28.7\left(\mathrm{CH}_{2}\right), 28.9\left(\mathrm{CH}_{2}\right), 39.3\left(\mathrm{PhCH}_{2}\right), 50.4$ $\left(\mathrm{CH}_{3}\right), 127.4$ (ArC), 129.0 (ArC), 129.8 (ArC), 135.9 $(\mathrm{ArC}), 170.7(\mathrm{C}=\mathrm{O}), 172.0(\mathrm{C}=\mathrm{O}), 175.2(\mathrm{C}=\mathrm{O})$. Anal Calcd for $\mathrm{C}_{13} \mathrm{H}_{15} \mathrm{NO}_{4}$ (249.26): C 62.58, H 6.01; Found: C: 61.33, H: 5.33 .

\section{4-Benzoylamino-4-oxobutyric Acid Methyl Ester (11a)}

Benzamide (2) $(1.23 \mathrm{~g}, 10 \mathrm{mmol})$ was reacted with succinic acid monomethyl ester chloride (7) (1.65 g, 12.26 $\mathrm{mmol})$ in toluene $(5 \mathrm{ml})$ according to general procedure $\mathrm{A}$ to afford $11 \mathrm{a}(1.06 \mathrm{~g}, 43 \%)$. IR $(\mathrm{KBr}) \tilde{v}=3291 \mathrm{~cm}^{-1}(\mathrm{~N}-\mathrm{H})$, $1740(\mathrm{C}=\mathrm{O}), 1709(\mathrm{C}=\mathrm{O}), 1677(\mathrm{C}=\mathrm{O}) .{ }^{1} \mathrm{H}$ NMR (200 $\left.\mathrm{MHz}, \mathrm{CDCl}_{3}\right): \delta=2.72\left(\mathrm{t}, J=4 \mathrm{~Hz}, 2 \mathrm{H}, \mathrm{CH}_{2}\right), 3.34(\mathrm{t}, J=4$ $\left.\mathrm{Hz}, 2 \mathrm{H}, \mathrm{CH}_{2}\right), 3.70$ (s, 3H, $\left.\mathrm{OCH}_{3}\right), 7.61(\mathrm{~m}, 3 \mathrm{H}, \mathrm{ArH}), 7.93$ $(\mathrm{m}, 2 \mathrm{H}, \mathrm{ArH}), 9.35(\mathrm{~s}, 1 \mathrm{H}, \mathrm{NH}) .{ }^{13} \mathrm{C} \mathrm{NMR}(50 \mathrm{MHz}$, $\left.\mathrm{CDCl}_{3}\right): \delta=28.6\left(\mathrm{CH}_{2}\right), 33.3\left(\mathrm{CH}_{2}\right), 52.3\left(\mathrm{OCH}_{3}\right), 128.3$ (ArC), 129.3 (ArC), 132.9 (ArC), 133.6 (ArC), 166.2 $(\mathrm{C}=\mathrm{O}), 173.4(\mathrm{C}=\mathrm{O}), 175.7(\mathrm{C}=\mathrm{O})$. Anal Calcd for $\mathrm{C}_{12} \mathrm{H}_{13} \mathrm{NO}_{4}$ (235.24): C 61.22, $\mathrm{H}$ 5.53; Found: C 61.23, H 5.45 .

4-(4-Hydroxybenzoylamino)-4-oxobutyric Acid Ethyl Ester (11c)

A solution of 4-benzyloxybenzamide $(500 \mathrm{mg}, 2.2 \mathrm{mmol})$ in dry THF $(10 \mathrm{ml})$ was treated with $\mathrm{NaH}(88 \mathrm{mg}, 2.2$ $\mathrm{mmol}$ ) and stirred for 1 hour at $20^{\circ} \mathrm{C}$. Fumaric acid monoethyl ester anhydride (600 mg, $2.2 \mathrm{mmol}$ ) was added and the mixture was heated overnight (procedure $B$ ). The product was purified by column chromatography on silica gel $\left(\mathrm{CH}_{2} \mathrm{Cl}_{2}\right)$ and crystallized from ether. A sample of the resulting benzyl ether $(150 \mathrm{mg})$ was dissolved in dry THF $(10 \mathrm{ml})$ and hydrogenated over Pd-C $(10 \%)$ for 3 hours. The reaction mixture was filtered and the solvent removed at reduced pressure. The residue was purified by column chromatography on silica gel $\left(\mathrm{CH}_{2} \mathrm{Cl}_{2}\right)$ to yield $11 \mathrm{c}(76 \mathrm{mg}$, $69 \%$, mp: $146 \sim 148^{\circ} \mathrm{C}$. IR $(\mathrm{KBr}) \tilde{v}=3091 \mathrm{~cm}^{-1}(\mathrm{~N}-\mathrm{H})$, $1715(\mathrm{C}=\mathrm{O}), 1675(\mathrm{C}=\mathrm{O}) .{ }^{1} \mathrm{H}$ NMR $\left(200 \mathrm{MHz}, \mathrm{CDCl}_{3}\right)$ : $\delta=1.31\left(\mathrm{t}, 3 \mathrm{H}, \mathrm{CH}_{3}\right), 2.76\left(\mathrm{t}, J=6 \mathrm{~Hz}, 2 \mathrm{H}, \mathrm{CH}_{2}\right), 3.78(\mathrm{t}$, $J=6 \mathrm{~Hz}, 2 \mathrm{H}, \mathrm{CH}_{2}$ ), 4.22 (q, 2H, $\mathrm{CH}_{2}$ ), 7.76 (d, $J=7 \mathrm{~Hz}, 2 \mathrm{H}$, $\operatorname{ArH}), 8.11$ (d, $J=7 \mathrm{~Hz}, 2 \mathrm{H}, \operatorname{ArH}), 8.62(\mathrm{~s}, 1 \mathrm{H}, \mathrm{NH}) .{ }^{13} \mathrm{C}$ NMR $\left(50 \mathrm{MHz}, \mathrm{CDCl}_{3}\right): \delta=14.1\left(\mathrm{CH}_{3}\right), 29.2\left(\mathrm{CH}_{2}\right), 29.6$
$\left(\mathrm{CH}_{2}\right), 61.7\left(\mathrm{CH}_{2}\right), 116.5$ (ArC), 117.0 (ArC), 125.9 (ArC), 128.8 (ArC), 129.7 (ArC), 161.1 (ArC), $168.2(\mathrm{C}=\mathrm{O})$, $172.3(\mathrm{C}=\mathrm{O}), 172.4(\mathrm{C}=\mathrm{O})$. Anal Calcd for $\mathrm{C}_{13} \mathrm{H}_{13} \mathrm{NO}_{5}$ (263.25): C 59.31, H 4.98; Found: C 59.38, H 4.95.

3-Oxo-3-phenylacetylaminopropionic Acid Methyl Ester $\underline{(12)}$

Phenylacetic acid amide (6a) $(1.0 \mathrm{~g}, 7.4 \mathrm{mmol})$ was reacted with malonic acid monomethyl ester chloride $(\mathbf{8})^{8)}$ $(1.22 \mathrm{~g}, 8.1 \mathrm{mmol})$ in toluene $(5 \mathrm{ml})$ to afford $12(520 \mathrm{mg}$, $30 \%$ ) after column chromatography (petroleum ether/ethyl acetate $3 / 1), \operatorname{mp~} 93 \sim 96^{\circ} \mathrm{C}$. IR $(\mathrm{KBr}) \tilde{v}=3266 \mathrm{~cm}^{-1}(\mathrm{~N}-\mathrm{H})$, $1750(\mathrm{C}=\mathrm{O}), 1735(\mathrm{C}=\mathrm{O}), 1698.2(\mathrm{C}=\mathrm{O}) .{ }^{1} \mathrm{H}$ NMR $\left(200 \mathrm{MHz}, \mathrm{CDCl}_{3}\right): \delta=3.76\left(\mathrm{~s}, 2 \mathrm{H}, \mathrm{CH}_{2}\right), 3.78(\mathrm{~s}, 3 \mathrm{H}$, $\left.\mathrm{OCH}_{3}\right), 3.85$ (s, 2H, $\left.\mathrm{ArCH}_{2}\right), 7.31$ (m, 3H, ArH), 7.40 (m, $2 \mathrm{H}, \mathrm{ArH}), 8.62$ (s, 1H, NH). ${ }^{13} \mathrm{C} \mathrm{NMR}\left(50 \mathrm{MHz}, \mathrm{CDCl}_{3}\right)$ : $\delta=44.2\left(\mathrm{CH}_{2}\right), 44.8\left(\mathrm{CH}_{2}\right), 53.1\left(\mathrm{OCH}_{3}\right), 128.3(\mathrm{ArC})$, 129.6 (ArC), 129.9 (ArC), 135.9 (ArC), $170.7(\mathrm{C}=\mathrm{O})$, $171.8(\mathrm{C}=\mathrm{O})$. Anal Calcd for $\mathrm{C}_{12} \mathrm{H}_{13} \mathrm{NO}_{4}$ : (235.24): $\mathrm{C}$ 61.22, H 5.53; Found: C 60.85, H 5.28.

\section{3-Methoxy- $N$-phenylacetylbenzamide (13)}

Phenylacetic acid amide (6a) $(1.35 \mathrm{~g}, 10 \mathrm{mmol})$ in dry toluene $(20 \mathrm{ml})$ was reacted with 3-methoxybenzoic acid chloride $(1.26 \mathrm{~g}, 9.4 \mathrm{mmol})$ as described in procedure $\mathrm{A}$ to afford imide 13 (926 mg, 35\%), mp: $112 \sim 114^{\circ} \mathrm{C}$. IR (KBr) $\tilde{v}=3312(\mathrm{~N}-\mathrm{H}) \mathrm{cm}^{-1}, 1709(\mathrm{C}=\mathrm{O}), 1683(\mathrm{C}=\mathrm{O}) .{ }^{1} \mathrm{H}$ NMR $\left(200 \mathrm{MHz}, \mathrm{CDCl}_{3}\right): \delta=3.86\left(\mathrm{~s}, 3 \mathrm{H}, \mathrm{OCH}_{3}\right), 4.36(\mathrm{~s}, 2 \mathrm{H}$, $\left.\mathrm{PhCH}_{2}\right), 7.17$ (t, J=2 Hz, 1H, ArH), 7.37 (m, 8H, ArH), $9.05(\mathrm{~s}, 1 \mathrm{H}, \mathrm{NH}) .{ }^{13} \mathrm{C} \mathrm{NMR}\left(50 \mathrm{MHz}, \mathrm{CDCl}_{3}\right): \delta=44.3$ $\left(\mathrm{CH}_{2}\right), 55.9\left(\mathrm{OCH}_{3}\right), 113.0(\mathrm{ArC}), 119.9$ (ArC), 120.2 (ArC), 127.6 (ArC), 129.0 (ArC), 130.2 (ArC), 130.4 (ArC), 134.1 (ArC), 134.4 (ArC), 160.4 (ArC), 167.7 $(\mathrm{C}=\mathrm{O}), 175.2(\mathrm{C}=\mathrm{O})$. Anal Calcd for $\mathrm{C}_{16} \mathrm{H}_{15} \mathrm{NO}_{3}(269.30)$ : C 71.29, H 5.57; Found: C 71.35, H: 5.54.

\section{4-Methoxy- $N$-phenylacetylbenzamide (14)}

Phenylacetic acid amide (6a) $(1.35 \mathrm{~g}, 10 \mathrm{mmol})$ was reacted with 4-methoxybenzoic acid chloride (1.26g, $9.4 \mathrm{mmol}$ ) as described in procedure $\mathrm{A}$ to afford imide 14 (807 mg, 30\%), mp: $162 \sim 164^{\circ} \mathrm{C}$. IR $(\mathrm{KBr}) \tilde{v}=3462 \mathrm{~cm}^{-1}$ $(\mathrm{N}-\mathrm{H}), 1786(\mathrm{C}=\mathrm{O}), 1601(\mathrm{C}=\mathrm{O}) .{ }^{1} \mathrm{H}$ NMR $(200 \mathrm{MHz}$, $\left.\mathrm{CDCl}_{3}\right): \delta=3.88\left(\mathrm{~s}, 3 \mathrm{H}, \mathrm{OCH}_{3}\right), 4.37\left(\mathrm{~s}, 2 \mathrm{H}, \mathrm{PhCH}_{2}\right), 6.95$ $(\mathrm{t}, J=5 \mathrm{~Hz}, 2 \mathrm{H}, \mathrm{ArH}), 7.37$ (m, 5H, ArH), 7.60 (d, $J=4 \mathrm{~Hz}$, 2H, ArH), 7.86 (d, $J=4 \mathrm{~Hz}, 2 \mathrm{H}, \mathrm{ArH}), 8.12$ (m, 5H, ArH), $9.45(\mathrm{~s}, 1 \mathrm{H}, \mathrm{NH}) .{ }^{13} \mathrm{C} \mathrm{NMR}\left(50 \mathrm{MHz}, \mathrm{CDCl}_{3}\right): \delta=39.3$ $\left(\mathrm{CH}_{2}\right), 56.0\left(\mathrm{OCH}_{3}\right), 114.2(\mathrm{ArC}), 125.8(\mathrm{ArC}), 127.4$ (ArC), 128.3 (ArC), 129.0 (ArC), 129.8 (ArC), 135.9 (ArC), $165.4(\mathrm{ArC}), 167.7(\mathrm{C}=\mathrm{O}), 170.7(\mathrm{C}=\mathrm{O})$. Anal Calcd for $\mathrm{C}_{16} \mathrm{H}_{15} \mathrm{NO}_{3}$ (269.30): C 71.29, H 5.57; Found: C 
5.03 .

4-( $N^{\prime}$-Benzoylhydrazino)-4-oxobut-2-enoic Acid Ethyl Ester (16)

The potassium salt of benzoylhydrazine was prepared by reaction of benzoylhydrazine hydrochloride (300 $\mathrm{mg}, 2.2$ $\mathrm{mmol})$ in a hot methanolic solution $(5 \mathrm{ml})$ of $\mathrm{KOH}(98 \mathrm{mg})$ and evaporation of the methanol at reduced pressure. The residue was dissolved in $\mathrm{CH}_{2} \mathrm{Cl}_{2}(10 \mathrm{ml})$ and fumaric acid chloride ethyl ester $(360 \mathrm{mg}, 2.7 \mathrm{mmol})$ was added. The solution was stirred at room temperature for 1 hour, the precipitate was filtered off and washed with cold $\mathrm{CH}_{2} \mathrm{Cl}_{2}$ to yield $16(256 \mathrm{mg}, 50 \%), \mathrm{mp}: 236 \sim 238^{\circ} \mathrm{C}$. IR $(\mathrm{KBr})$ $\tilde{v}=3498 \mathrm{~cm}^{-1}(\mathrm{~N}-\mathrm{H}), 3390(\mathrm{~N}-\mathrm{H}), 1654(\mathrm{C}=\mathrm{O}), 1650$ $(\mathrm{C}=\mathrm{O}) .{ }^{1} \mathrm{H}$ NMR $(200 \mathrm{MHz}, \mathrm{DMSO}): \delta=1.26(\mathrm{t}, 3 \mathrm{H}$, $\left.\mathrm{CH}_{3}\right), 4.20$ (q, 2H, $\mathrm{CH}_{2}$ ), 6.72 (d, $\left.J=15 \mathrm{~Hz}, 1 \mathrm{H}, \mathrm{CH}\right), 7.09$ (d, $J=15 \mathrm{~Hz}, 1 \mathrm{H}, \mathrm{CH}), 7.58(\mathrm{~m}, 3 \mathrm{H}, \mathrm{ArH}), 7.92(\mathrm{~m}, 2 \mathrm{H}$, $\mathrm{ArH}), 10.53(\mathrm{~s}, 1 \mathrm{H}, \mathrm{NH}), 11.15$ (s, 1H, NH). ${ }^{13} \mathrm{C} \mathrm{NMR}$ (50 MHz, DMSO): $\delta=14.9\left(\mathrm{CH}_{3}\right), 61.7\left(\mathrm{CH}_{2}\right), 128.3$ (ArC), 129.4 (ArC), 130.8 (ArC), $131.5(\mathrm{ArC}), 132.7$ $(\mathrm{ArC}), 133.4(\mathrm{ArC}), 135.4(\mathrm{CH}), 137.5(\mathrm{CH}), 161.4$ $(\mathrm{C}=\mathrm{O}), 165.6(\mathrm{C}=\mathrm{O}), 166.7(\mathrm{C}=\mathrm{O})$. Anal Calcd for $\mathrm{C}_{13} \mathrm{H}_{14} \mathrm{~N}_{2} \mathrm{O}_{4}$ (262.26) C: 59.54, H: 5.38; Found: C 59.48, H 5.34.

4-Benzoylaminoxy-4-oxobut-2-enoic Acid Ethyl Ester (18)

A mixture of benzoic acid $(6.10 \mathrm{~g}, 50 \mathrm{mmol})$, hydroxylamine $(65 \mathrm{mmol})$ and dicyclohexyl carbodiimide $(10.30 \mathrm{~g}$, $50 \mathrm{mmol})$ in methanol $(30 \mathrm{ml})$ was stirred for 1 hour. The methanol was evaporated at reduced pressure and the residue extracted with $10 \%$ aqueous $\mathrm{NaOH}(10 \mathrm{ml})$. The basic phase was acidified with $10 \% \mathrm{HCl}(10.3 \mathrm{ml})$ and extracted with $\mathrm{CH}_{2} \mathrm{Cl}_{2}(30 \mathrm{ml})$. The organic phase was dried $\left(\mathrm{Na}_{2} \mathrm{SO}_{4}\right)$ and the solvent removed at reduced pressure. $\mathrm{A}$ sample of this crude hydroxamic acid $17(250 \mathrm{mg})$, fumaric acid ethyl ester (3a) $(300 \mathrm{mg}, 1.8 \mathrm{mmol})$, and triethylamine $(184 \mathrm{mg}, 1.8 \mathrm{mmol})$ was dissolved in toluene $(20 \mathrm{ml})$ and stirred for 1 hour at room temperature to yield $18(290 \mathrm{mg}$, $61 \%$ after column chromatography on silica gel, $\mathrm{mp}$ : $76 \sim 78^{\circ} \mathrm{C}$. IR $(\mathrm{KBr}): \tilde{v}=3076 \mathrm{~cm}^{-1}(\mathrm{~N}-\mathrm{H}), 1796(\mathrm{C}=\mathrm{O})$, $1720(\mathrm{C}=\mathrm{O}), 1634(\mathrm{C}=\mathrm{O}) .{ }^{1} \mathrm{H}$ NMR $\left(200 \mathrm{MHz}, \mathrm{CDCl}_{3}\right)$ : $\delta=1.28\left(\mathrm{t}, 3 \mathrm{H}, \mathrm{CH}_{3}\right), 4.24\left(\mathrm{q}, 2 \mathrm{H}, \mathrm{CH}_{2}\right), 6.84(\mathrm{~d}, J=13 \mathrm{~Hz}$, $1 \mathrm{H}, \mathrm{CH}), 6.98$ (d, $J=13 \mathrm{~Hz}, 1 \mathrm{H}, \mathrm{CH}), 7.48(\mathrm{~m}, 2 \mathrm{H}, \mathrm{ArH})$, 7.59 (m, 1H, ArH), 8.09 (m, 2H, ArH), $9.13(\mathrm{~s}, 1 \mathrm{H}, \mathrm{NH})$. ${ }^{13} \mathrm{C}$ NMR $\left(50 \mathrm{MHz}, \mathrm{CDCl}_{3}\right): \delta=14.4\left(\mathrm{CH}_{3}\right), 61.9\left(\mathrm{CH}_{2}\right)$, 128.8 (ArC), 129.3 (ArC), 130.5 (ArC), 131.7 (ArC), 132.4 $(\mathrm{ArC}), 133.5(\mathrm{ArC}), 135.5(\mathrm{CH}), 137.5(\mathrm{CH}), 162.7$ $(\mathrm{C}=\mathrm{O}), \quad 169.8(\mathrm{C}=\mathrm{O}), 172.3(\mathrm{C}=\mathrm{O})$. Anal Calcd for $\mathrm{C}_{13} \mathrm{H}_{13} \mathrm{NO}_{5}$ (263.25): C 59.31, H 4.98; Found: $\mathrm{C} 60.03, \mathrm{H}$
3-(N'-Phenylhydrazinocarbonyl)-acrylic Acid Ethyl Ester (20a)

A mixture of phenylhydrazine $(1.08 \mathrm{~g}, 10 \mathrm{mmol})$ and fumaric acid chloride ethyl ester $(1.34 \mathrm{~g}, 10 \mathrm{mmol})$ in dichloromethane $(25 \mathrm{ml})$ and $2 \sim 3$ drops of triethyl amine was stirred for 30 minutes. The precipitate was filtered off and washed with $\mathrm{CH}_{2} \mathrm{Cl}_{2}$ to yield 20a (976 mg, 48\%), mp: $140 \sim 142^{\circ} \mathrm{C}$. IR $(\mathrm{KBr}): \tilde{v}=3368 \mathrm{~cm}^{-1}(\mathrm{~N}-\mathrm{H}), 3096(\mathrm{~N}-\mathrm{H})$, $1644(\mathrm{C}=\mathrm{O}), 1614(\mathrm{C}=\mathrm{O}) .{ }^{1} \mathrm{H}$ NMR $\left(200 \mathrm{MHz}, \mathrm{CDCl}_{3}\right)$ : $\delta=1.27 \delta\left(\mathrm{t}, J=7 \mathrm{~Hz}, 3 \mathrm{H}, \mathrm{CH}_{3}\right), 4.22(\mathrm{q}, J=7 \mathrm{~Hz}, 2 \mathrm{H}$, $\left.\mathrm{CH}_{2}\right), 6.77$ (d, J=14 z, 1H, CH), $6.96(\mathrm{~m}, 2 \mathrm{H}, \mathrm{ArH}), 7.27$ (d, $J=14 \mathrm{~Hz}, 1 \mathrm{H}, \mathrm{CH}$ ), 7.49 (m, $J=8 \mathrm{~Hz}, 3 \mathrm{H}, \mathrm{ArH}), 9.98$ (s, $1 \mathrm{H}, \mathrm{NH}), 10.15(\mathrm{~s}, 1 \mathrm{H}, \mathrm{NH}) .{ }^{13} \mathrm{C}$ NMR $\left(50 \mathrm{MHz}, \mathrm{CDCl}_{3}\right)$ : $\delta=13.8\left(\mathrm{CH}_{3}\right), 61.4\left(\mathrm{OCH}_{2}\right), 113.2(\mathrm{ArC}), 113.3(\mathrm{ArC})$, 119.1 (ArC), $130.0(\mathrm{ArC}), 131.2(\mathrm{ArC}), 135.1(\mathrm{CH}), 137.8$ (CH), 144.6 (ArC), $165.2(\mathrm{C}=\mathrm{O}), 166.4(\mathrm{C}=\mathrm{O})$. Anal Calcd for $\mathrm{C}_{12} \mathrm{H}_{14} \mathrm{~N}_{2} \mathrm{O}_{3}$ (234.25): C 61.53, H 6.02; Found: C $61.60,6.08$.

3-( $N^{\prime}$-(2,4-Dinitrophenyl)-hydrazinocarbonyl]-acrylic Acid Ethyl Ester (20b)

A mixture of 2,4-dinitrophenylhydrazine $(1.5 \mathrm{~g}, 7.6$ $\mathrm{mmol})$ and fumaric acid chloride ethyl ester $(1.13 \mathrm{~g}, 8.4$ $\mathrm{mmol})$ in dichloromethane $(30 \mathrm{ml})$ and $2 \sim 3$ drops of triethyl amine was stirred for 30 minutes. The precipitate was filtered off, and washed with $\mathrm{CH}_{2} \mathrm{Cl}_{2}$ to yield 20b (604 mg, 78\%), mp: $161 \sim 163^{\circ} \mathrm{C}$. IR (KBr) $\tilde{v}=3278 \mathrm{~cm}^{-1}$ $(\mathrm{N}-\mathrm{H}), 3024(\mathrm{~N}-\mathrm{H}), 1695(\mathrm{C}=\mathrm{O}), 1670(\mathrm{C}=\mathrm{O}) .{ }^{1} \mathrm{H}$ NMR $\left(200 \mathrm{MHz}, \mathrm{CDCl}_{3}\right): \delta=1.28 \delta\left(\mathrm{t}, J=7 \mathrm{~Hz}, 3 \mathrm{H}, \mathrm{CH}_{3}\right), 4.24$ (q, $\left.J=7 \mathrm{~Hz}, 2 \mathrm{H}, \mathrm{CH}_{2}\right), 6.75(\mathrm{~d}, J=14 \mathrm{~Hz}, 1 \mathrm{H}, \mathrm{CH}), 7.16$ (d, $J=14 \mathrm{~Hz}, 1 \mathrm{H}, \mathrm{CH}), 7.56(\mathrm{~s}, 1 \mathrm{H}, \mathrm{ArH}), 8.37$ (m, 2H, ArH). $10.08(\mathrm{~s}, 1 \mathrm{H}, \mathrm{NH}), 10.28(\mathrm{~s}, 1 \mathrm{H}, \mathrm{NH}) .{ }^{13} \mathrm{C}$ NMR $(50 \mathrm{MHz}$, $\left.\mathrm{CDCl}_{3}\right): \delta=14.8\left(\mathrm{CH}_{3}\right), 61.8\left(\mathrm{OCH}_{2}\right), 110.0(\mathrm{ArC}), 113.5$ (ArC), $134.4(\mathrm{ArC}), 135.2(\mathrm{CH}), 137.6(\mathrm{CH}), 143.5(\mathrm{ArC})$, 148.9 (ArC), $150.1(\mathrm{ArC}), 165.0(\mathrm{C}=\mathrm{O}), 166.1(\mathrm{C}=\mathrm{O})$. Anal Calcd for $\mathrm{C}_{12} \mathrm{H}_{12} \mathrm{~N}_{4} \mathrm{O}_{7}$ (324.25): $\mathrm{C}$ 44.45, $\mathrm{H}$ 3.73; Found: C 44.36, 3.69.

3-(N'-Phenyl)-hydrazinocarbonyl-acrylic Acid Octyl Ester $\underline{(20 c)}$

A solution of phenylhydrazine $(504 \mathrm{mg}, 4.6 \mathrm{mmol})$ and fumaric acid monooctyl ester anhydride $(1.15 \mathrm{~g}, 5.0 \mathrm{mmol})$ in $\mathrm{CH}_{2} \mathrm{Cl}_{2}(10 \mathrm{ml})$ and $.2 \sim 3$ drops of triethyl amine was stirred at room temperature for 1 hour. The solution was cooled to $0^{\circ} \mathrm{C}$, the precipitate was filtered off and washed with cold $\mathrm{CH}_{2} \mathrm{Cl}_{2}$ to yield hydrazide $20 \mathrm{c}(800 \mathrm{mg}, 57 \%)$, $\mathrm{mp}: 130 \sim 132^{\circ} \mathrm{C}$. IR $(\mathrm{KBr}): \tilde{v}=3383 \mathrm{~cm}^{-1}(\mathrm{~N}-\mathrm{H}), 3283$ $(\mathrm{N}-\mathrm{H}), 1675(\mathrm{C}=\mathrm{O}), 1594(\mathrm{C}=\mathrm{O}) .{ }^{1} \mathrm{H}$ NMR $(200 \mathrm{MHz}$, 
$\left.\mathrm{CDCl}_{3}\right): \delta=0.91\left(\mathrm{t}, 3 \mathrm{H}, \mathrm{CH}_{3}\right), 1.32\left(\mathrm{~m}, 10 \mathrm{H}, 5 \times \mathrm{CH}_{2}\right), 4.23$ (t, $\left.J=4 \mathrm{~Hz}, 2 \mathrm{H}, \mathrm{CH}_{2}\right), 6.73(\mathrm{~d}, J=14 \mathrm{~Hz}, 1 \mathrm{H}, \mathrm{CH}), 7.01$ (d, $2 \mathrm{H}, \mathrm{ArH}), 7.21$ (d, $J=14 \mathrm{~Hz}, 1 \mathrm{H}, \mathrm{CH}), 7.51$ (m, 3H, ArH), $9.88(\mathrm{~s}, 1 \mathrm{H}, \mathrm{NH}), 10.11(\mathrm{~s}, 1 \mathrm{H}, \mathrm{NH}) .{ }^{13} \mathrm{C} \mathrm{NMR}(50 \mathrm{MHz}$, $\left.\mathrm{CDCl}_{3}\right): \delta=13.9\left(\mathrm{CH}_{3}\right), 22.8\left(\mathrm{CH}_{2}\right), 26.1\left(\mathrm{CH}_{2}\right), 29.8$ $\left(\mathrm{CH}_{2}\right), 30.2\left(\mathrm{CH}_{2}\right), 30.5\left(\mathrm{CH}_{2}\right), 32.1\left(\mathrm{CH}_{2}\right), 65.4\left(\mathrm{OCH}_{2}\right)$, 112.9 (ArC), 118.7 (ArC), 129.1 (ArC), $133.8(\mathrm{CH}), 134.0$ $(\mathrm{CH}), 134.2(\mathrm{ArC}), 165.0(\mathrm{C}=\mathrm{O}), 165.7(\mathrm{C}=\mathrm{O})$. Anal Calcd for $\mathrm{C}_{12} \mathrm{H}_{12} \mathrm{~N}_{4} \mathrm{O}_{7}$ (324.25): $\mathrm{C} 68.65, \mathrm{H}$ 8.49; Found: $\mathrm{C}$ $68.05,8.43$.

\section{(2,5-Dioxo-4-phenylpyrrolidin-3-ylidene)-acetic Acid} Methyl Ester (24)

4-Phenylpyrrolidine-2,3,5-trione (23) was prepared by sodium ethoxide-catalyzed condensation of phenylacetamide (22) and ethyl oxalate (21) as described in the literature. $^{3)}$ A solution of $24(94.5 \mathrm{mg}, 0.5 \mathrm{mmol})$ and methoxycarbonyl-methylenetriphenyl phosphorane (167 $\mathrm{mg}, 0.5 \mathrm{mmol})$ in dichloromethane $(30 \mathrm{ml})$ was refluxed for 3 hours. The solvent was removed under reduced pressure and the crude product purified by column chromatography on silica gel to yield 24 (84 mg, 69\%), mp: $92 \sim 93^{\circ} \mathrm{C} .{ }^{1} \mathrm{H}-$ NMR (200 MHz, $\left.\mathrm{CDCl}_{3}\right): \delta=3.52\left(\mathrm{~s}, 3 \mathrm{H}, \mathrm{OCH}_{3}\right), 4.69$ (s, $1 \mathrm{H}, \mathrm{PhCH}), 6.70(\mathrm{~s}, 1 \mathrm{H}, \mathrm{CH}), 7.37$ (m, 5H, ArH). ${ }^{13} \mathrm{C}-$ NMR (50 MHz, $\left.\mathrm{CDCl}_{3}\right): \delta=53.9\left(\mathrm{CH}_{3}\right), 125.8(\mathrm{CH}), 128.1$ (ArC), 130.6 (ArC), 130.8 (ArC), 134.0 (ArC), 134.2 (ArC), $165.3(\mathrm{C}=\mathrm{O}), 166.3(\mathrm{C}=\mathrm{O}), 170.7(\mathrm{C}=\mathrm{O})$. Anal

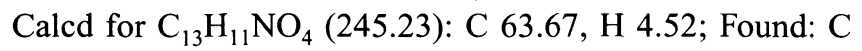
$62.75, \mathrm{H} 4.81$.

\section{(2,5-Dioxo-4-phenylpyrrolidin-3-ylidene)-acetic Acid}

\section{Ethyl Ester (25)}

A solution of 4-phenylpyrrolidine-2,3,5-trione (23) (510 $\mathrm{mg}, 2.7 \mathrm{mmol}$ ) and ethoxycarbonyl-methylene-triphenyl phosphorane (940 $\mathrm{mg} 2.7 \mathrm{mmol}$ ) in dichloromethane $(30 \mathrm{ml})$ was refluxed for 3 hours. The solvent was removed under reduced pressure and the crude product was purified by column chromatography on silica gel to afford ester $\mathbf{2 7}$ (386 mg, 55\%), mp: $108 \sim 109^{\circ} \mathrm{C} .{ }^{1} \mathrm{H}-\mathrm{NMR}(200 \mathrm{MHz}$, $\mathrm{CDCl}_{3}$ ): $\delta=1.04\left(\mathrm{t}, J=7.1 \mathrm{~Hz}, 3 \mathrm{H}, \mathrm{CH}_{3}\right.$ ), 4.01 (q, $J=7.1$ $\mathrm{Hz}, 2 \mathrm{H}, \mathrm{OCH}_{2}$ ), 6.95 (s, 1H, CH), 7.66 (m, 5H, ArH). Anal Calcd for $\mathrm{C}_{14} \mathrm{H}_{13} \mathrm{NO}_{4}$ (259.26): C 64.86, H 5.05; Found: C 63.92, H 5.36.

\section{3-Methoxymethylene-4-phenylpyrrolidine-2,5-dione (26)}

A solution of methoxymethylene-triphenylphosphonium chloride $(911 \mathrm{mg}, 2.7 \mathrm{mmol})$ in dry THF was treated at $-78^{\circ} \mathrm{C}$ with potassium tert-butoxide $(303 \mathrm{mg}, 2.7 \mathrm{mmol})$. The solution was allowed to warm to $20^{\circ} \mathrm{C}$, 4phenylpyrrolidine-2,3,5-trione (23) (510 $\mathrm{mg}, 2.7 \mathrm{mmol})$ was added, and stirring was continued for 24 hours. The solvent was removed under reduced pressure and the residue was purified by column chromatography on silica gel (dichloromethane/methanol $=9 / 1$ ) to afford the enol ether 26 (283 mg, 48\%). ${ }^{1} \mathrm{H}-\mathrm{NMR}\left(200 \mathrm{MHz}, \mathrm{CDCl}_{3}\right): \delta=3.51$ (s, 3H, $\left.\mathrm{OCH}_{3}\right), 4.16(\mathrm{~s}, 1 \mathrm{H}, \mathrm{PhCH}), 5.25(\mathrm{~s}, 1 \mathrm{H}, \mathrm{CH}), 7.37$ (m, 5H, ArH). Anal Calcd for $\mathrm{C}_{12} \mathrm{H}_{11} \mathrm{NO}_{3}$ (217.22): $\mathrm{C}$ 66.35, H 5.10; Found: C 65.39, H 4.86.

3-(2,5-Dioxo-4-phenylpyrrolidin-3-ylidene)-propionic Acid Ethyl Ester (27)

A solution of ethoxycarbonyl-ethylene-triphenylphosphorane bromide in dry THF $(20 \mathrm{ml})$ was treated at $-78^{\circ} \mathrm{C}$ with tert-BuOK $(303 \mathrm{mg}, 2.7 \mathrm{mmol})$. After 15 minutes stirring, trione $23(500 \mathrm{mg}, 2.7 \mathrm{mmol})$ was added. The mixture was allowed to warm to room temperature and stirring was continued for 24 hours. The solvent was removed under reduced pressure. The residue was redissolved in $\mathrm{CH}_{2} \mathrm{Cl}_{2}$ and filtered and the product was purified by column chromatography on silica gel (dichloromethane/methanol 9/1) to yield the ester 27 (295 mg, 40\%). 'H-NMR (200 MHz, $\left.\mathrm{CDCl}_{3}\right): \delta=1.11$ (t, $\left.J=7.1 \mathrm{~Hz}, 3 \mathrm{H}, \mathrm{CH}_{3}\right), 3.76\left(\mathrm{~m}, 2 \mathrm{H}, \mathrm{CH}_{2}\right), 4.13(\mathrm{q}, J=7.1 \mathrm{~Hz}$, $\left.2 \mathrm{H}, \mathrm{OCH}_{2}\right), 6.86(\mathrm{~s}, 1 \mathrm{H}, \mathrm{CH}), 7.66(\mathrm{~m}, 5 \mathrm{H}, \mathrm{ArH})$. Anal Calcd for $\mathrm{C}_{15} \mathrm{H}_{15} \mathrm{NO}_{4}$ (273.28): C 65.92, H 5.53; Found: $\mathrm{C}$ 64.12, H 5.05 .

Acknowledgement

The authors would like to thank BASF AG for support and for testing the compounds for their fungicidal activity. Special thanks go to Dr. RHeinheimer and Dr. SPEAKMAN for supervising the biological tests.

\section{References}

1) Krohn, K.; C. Franke, P. G. Jones, H.-J. Aust, S. Draeger \& B. Schulz: Wirkstoffe aus Pilzen, I. Isolierung, Synthese und biologische Wirkung von Coniothyriomycin und analogen offenkettigen Imiden. Liebigs Ann. Chem.: 789 798, 1992

2) Suhara, Y.; H. B. Maruyama, Y. Кotoh, Y. Miyasaka, K. Yokose, H. Shirai \& K. TAKano: New antibiotic, fumaramidmycin. II. Isolation, structure, and synthesis. J. Antibiotics 28: 648 655, 1975

3) Skinner, G. S. \& C. B. Miller, Jr.: On the structure of pyrrolidinetriones and oxazolidines. J. Am. Chem. Soc. 75: 977 998, 1953

4) Poschenrieder, H. \& H.-D. Stachel: Synthesis of pyrrolo[3,4-c]pyridazines. J. Heterocyclic Chem. 32: $1457 \sim 1460,1995$

5) Krohn, K.; A. Michel, U. Flörke, H.-J. Aust, S. Draeger \& B. SChulz: Biologically active metabolites from fungi, 4. Palmarumycins $\mathrm{CP}_{1}$ to $\mathrm{CP}_{4}$ from 
Coniothyrium palmarum: Isolation, structure elucidation and biological activity. Liebigs Ann. Chem.: 1093 1097, 1994

6) LaXmi, Y. S. \& D. S. IYengar: Synthesis and reactions of 5-benzylidene-2-phenyl-4,5-dihydro-1,3-oxazol-4-ones. J. Chem. Soc. Perkin Trans. I: 3043 3045, 1995
7) CASON, J. \& J. B. Miller: Branched-chain fatty acids. I. Synthesis of 17-methyloctadecanoic acid. J. Am. Chem. Soc. 64: 1106 1110, 1942

8) Scheepens, P. C. \& H. Fehrmann: Cultivation of Phytophthora infestans on defined nutrient media. Phytopath. Z. 93: 126 136, 1978 